0028-3932(95)00067-4

\title{
THE COGNITIVE NEUROSCIENCE OF MENTAL IMAGERY
}

\author{
S. M. KOSSLYN, ${ }^{*} †$ M. BEHRMANN $\ddagger$ and M. JEANNEROD \\ †Department of Psychology, Harvard University, Cambridge, Massachusetts, U.S.A.; \\ $\ddagger$ Department of Psychology, Carnegie Mellon University, Pittsburgh, Pennsylvania \\ and §̧Vision et Motricité, 16, avenue du Doyen Lépine, 69500 Bron, France
}

(Received 12 November 1994; accepted 10 January 1995)

This special issue is devoted to recent work on mental imagery. To get a sense for the subject matter, answer the following questions: How many windows are there in your living room? What socks did you wear yesterday? Is there room in your refrigerator for a litre of milk? These questions typically evoke visual mental imagery. For example, when answering the first, people usually visualize the room, and then 'scan' over each wall, 'seeing' each window with their mind's eye. Similarly, if asked whether a cat's meow has a higher pitch than the sound of a blender, most people report 'hearing' the sounds in their 'mind's ear'. Such tasks evoke auditory mental imagery. Visual mental imagery is 'seeing' in the absence of the appropriate immediate sensory input, auditory mental imagery is 'hearing' in the absence of the appropriate immediate sensory input, and so on. Imagery is distinct from perception, which is the registration of physically present stimuli.

The examples illustrate that imagery plays an important role in memory and spatial reasoning, but as we shall see, these functions merely scratch the surface. Imagery also plays a role in abstract reasoning, skill learning, and language comprehension. One of the major insights about imagery is that - even within a single sensory modality - it is not a single, undifferentiated ability. Rather, as the opening example suggests, imagery involves a host of processes working together. The image of one's living room must be formed at the outset, it then must be scanned and interpreted, and must be maintained while this is occurring. Moreover, in many situations the imaged object must be transformed. For example, when asked whether frogs have stubby green tails, people often report 'mentally rotating' the amphibian and 'looking' at its posterior quarters.

Interest in mental imagery has waxed and waned for centuries, and is now undergoing a resurgence. Unlike previous periods of enthusiasm for the topic, the present one is not driven by philosophical concerns or claims based on introspection. Rather, the present

\footnotetext{
*Address for reprint requests: Department of Psychology, Harvard University, 33 Kirkland Street, Cambridge, MA 02138, U.S.A.
} 
interest has been fueled by the emergence of new methods and new ways to conceptualize imagery. As illustrated in this special issue, the confluence of these factors has produced a new wave of discoveries not only about the nature and function of imagery, but also about its neural bases. Indeed, because imagery has been shown to share mechanisms with likemodality perception, theories of imagery have been able to stand on the shoulders of theories of perception. This state of affairs has resulted in the somewhat ironic fact that an area that was considered beyond the pale 20 years ago is now of the first cognitive domains to be firmly rooted in the brain.

Interest in imagery can be traced back at least to the time of Plato, who thought that memories were based on images; on his view, memories are carved into the mind much like pictures can be carved on a wax tablet. He even considered individual differences, likening them to differences in the purity of the wax and in the ease of carving figures into it. Interest in imagery continued on for centuries. For example, the British Associationists conceived of thought itself as sequences of mental images, and the original psychologies of Wundt and James placed considerable emphasis on imagery.

Imagery fell from grace, however, when psychology became obsessed with method. The behaviorists stressed that the scientific method requires that one study phenomena that are publicly accessible. This, of course, was not what was going on in other sciences, such as physics-which were quite happy to draw inferences about unobservable phenomena by studying their 'footprints' (e.g. tracks in cloud chambers). But more than this, the behaviorists argued effectively that it was not clear how to conceive of mental images. Pictures in the head? Who looks at them? In retrospect, it seems that the eclipse of research on imagery was a result of three events: A lack of sophisticated methodologies for studying mental events, impoverished theoretical constructs, and the apparent success of the behaviorist approach. This last factor was critical because it justified a shift in the way the field of psychology was characterized. Whereas James conceived of psychology as the study of mental life, Watson declared that it should be the study of behavior. There is no place for mental events, let alone those that do not have distinctive behavioral sequellae, in a field that studies behavior qua behavior.

Imagery came into its own again in the early 1970s, riding the tide of the "cognitive revolution' [see 10]. As the limitations of behaviorism became apparent, researchers once again became receptive to theorizing about internal events. Three kinds of work emerged from the application of the methods of cognitive psychology to the study of imagery. First, Paivio and his collaborators demonstrated that people can learn and remember highly imageable materials better than abstract materials [e.g. see 16]. Second, Segal and her colleagues used signal detection paradigms to show that imagery interferes more with likemodality perception than with different-modality perception (e.g. visual imagery interferes with visual perception more than with auditory perception and vice versa for auditory imagery [18]). And third, Shepard and his collaborators [e.g. 21] demonstrated that images act as 'surrogate objects' during reasoning. For example, when subjects compared two objects (e.g. three-dimensional block figures) to determine whether they had the same shape or were mirror images, they required more time when the objects had to be "mentally rotated' greater amounts to be aligned. In spite of the fact that images are not actual objects that must obey the laws of physics, objects in images often behave like actual objects.

A second innovation in imagery research took place during the $1970 \mathrm{~s}$, but this one was conceptual, not methodological. Imagery was conceptualized as a type of data structure, 
which was embedded in an information processing system [e.g. 2, 12]. This perspective led to a host of experiments that were designed to reveal structural properties of images as specific types of data structures. A debate ensued about this issue, which quickly bogged down when researchers realized the limitations of purely behavioral approaches [e.g. see 1].

The difficulties in characterizing the way images are represented and processed set the stage for the most recent development in this field: The cognitive neuroscience approach. Researchers began to use neuropsychological data to inform theories of the structure of the processing system [e.g. 7]. And more recently, such data have been used to characterize the nature of the representation itself [e.g. 13].

As each new approach has matured, it has supplemented - not replaced-the earlier approaches. Thus, new developments have fleshed out the field, which can now be characterized as encompassing a number of relatively distinct domains.

\section{RESEARCH DOMAINS}

The study of imagery has now developed into a field in its own right. In this section we briefly review the major areas of research, which we have grouped into four major categories: Learning and memory; perception and action; information processing; and reasoning. In all cases, researchers began by characterizing the function itself, and only then sought the neural bases of these functions. We do not believe that it is wise to treat the neuropsychology of imagery as a distinct field or area; rather, it is an integral part of the study of imagery more generally. Neuropsychological findings and theories illuminate the nature of imagery representations and processes, as well as the functions of imagery. We seek to avoid the trap that has befallen some parts of developmental psychology, where theories of the development of cognitive function make little contact with more sophisticated theories of those functions that have been formulated by cognitive scientists.

\section{Learning and memory}

We can distinguish three roles for imagery in learning and memory.

Imagery and learning. The ancient Greeks discovered that one's memory for a set of objects could be greatly enhanced if one visualized them interacting in some way. Once one has visualized a scene and encoded it into memory, it then can be recalled in an image. For example, if you want to remember a set of errands, you can visualize yourself doing them in order. Later you can visualize the first part of the sequence, and 'see' yourself progress through the entire sequence, which will help you to recall the entire set. Bower [4] showed that much of the mnemonic power of imagery comes from its ability to represent associations between distinct objects.

Imagery and memory. Even if they did not set out to store images intentionally, people often will use imagery later to recall information. Imagery is used spontaneously to recall the shape, color, size, and texture of objects or spatial relations in scenes that: (a) have been considered relatively infrequently, and (b) cannot easily be inferred from facts associated with a superordinate category or from verbal material. For example, imagery is used to determine whether a German Shepherd dog's ears protrude above the top of its skull, but not whether it has four legs [see 14].

Using imagery to learn skills. If one visualizes oneself performing a skill one is acquiring, one will actually improve at the skill. For example, if you were learning a new dance, 
visualizing the steps would lead you to be a better dancer-provided that imagined practice is intermixed with actual practice [e.g. see 17].

\section{Perception and action}

Imagery draws on mechanisms used not only in perception, but also in motor control. Shared mechanism with perception. It is not an accident that we speak of 'seeing' objects in visual images, or 'hearing' sounds in auditory images. The phenomenology of mental imagery is in many ways like that of like-modality perception. This fact has suggested to many that the same mechanisms are involved in at least some aspects of imagery and likemodality perception. There is now a large literature attesting to the validity of this inference [e.g. see 9]. For example, Craver-Lemley and Reeves [5] showed that visualizing a line interferes with the ability to see how two line segments are aligned, which is as expected if imagery engages mechanisms that otherwise would be used in perception. It is likely that imagery processes actually play a role in perception, especially when input is degraded - in which cases imagery may help to complete 'noisy' input [see 13].

Such findings are consistent with several important features of the neuroanatomy of the visual system, such as the fact that virtually every visual area that sends information to another area also receives fibers from that other area-and the fibers running in each direction are of comparable size [e.g. see 8]. Moreover, various brain scanning techniques have revealed that visual areas in the occipital, temporal, and parietal lobes are also activated during visual mental imagery [for a review, see 13]. In addition, damage to portions of the brain used in visual perception often also result in corresponding deficits in visual imagery [e.g. see 15; for an interesting exception, see 3].

Motor control. Georgopoulos and his colleagues [11] have shown that some forms of image transformation are accompanied by activity in the motor cortex. These transformations involve anticipating where to reach, but the motor system may play a role more generally in image transformations. Indeed, Deutsch et al. [6] found that the right hemisphere, particularly the parietal and frontal lobes, are particularly active when subjects perform visual mental rotation; these regions of cortex include several areas that play key roles in motor control. Thus, it is possible that the motor system plays an important role in imagery, specifically in 'image transformation' processes, as noted below.

\section{Information processing}

Images can be regarded as a particular way in which information is stored, and these representations are processed in specific ways. The system in which images are processed can be conceptualized in terms of four major abilities, as noted below. Although this approach has been worked out in the most detail for visual mental images, corresponding distinctions may also exist in other modalities.

Image inspection. Image inspection is the ability to extract information that is depicted in an image. For example, one can determine whether the first three notes of "Three blind mice" go up or down, or whether there are any curved lines in the uppercase letter ' $b$ '. If one could not 'inspect' patterns in images, images could not serve as repositories of information. In the course of inspecting imaged objects, subjects report having to 'zoom in' to 'see' small details, and in fact, people require more time to evaluate objects imaged at small sizes than to evaluate objects imaged at larger sizes. Similarly, images can only be so large before they appear to 'overflow' one's field of view. In addition, the farther one must 
shift one's mental gaze to 'see' a portion of an imaged object, the more time is taken. And some parts of objects are more difficult to 'see' in images; for example, a parallelogram is harder to 'see' in an image of the Star of David than is a triangle [for reviews of such findings, see 13].

Image generation. Logically, images can arise in only two ways: First, sensory input can be retained (over the course of a few seconds, not simply a fraction of a second); second, information stored in long-term memory can be activated. Most imagery appears to arise when information in long-term memory is activated. Indeed, many images are novel combinations of objects or characteristics that were encoded at different times and places. For example, most people can visualize their favorite politician riding a donkey, and report whether he or she could see over the top of the animal's head-even though such a scene was never witnessed. Many studies have now shown that such 'image generation' involves serial processing; indeed, the time to form images typically increases linearly for each additional part or property of the to-be-visualized object, and the time to visualize a given part often can be predicted by the order in which subjects typically draw the parts. Image, generation has been the topic of intensive study in neuropsychology, particularly since Farah [7] reviewed the case study literature and claimed that this ability is selectively disrupted by damage to the posterior left cerebral hemisphere.

Image transformation. Imagery would be of limited value if we could only recall past events or recombine these memories in specific ways. In many cases we want to 'turn something over' in our minds [see 19]. One of the most interesting facts about image transformations is that they often preserve the time course of the corresponding actual transformation. For example, if you rotated the upper case letter $n 90^{\circ}$ clockwise, would it be another letter? What if you rotated an upper case letter $p 180^{\circ}$ ? The farther you would have to rotate the object, more time you would require. Similarly, the more squares one must fold to visualize a set of connected squares forming a box, the more time is required [20]. Such results are as expected if one is simulating what would occur if an object were actually manipulated in a specific way, and-as noted above- - the motor system may play a role in image transformations.

Image retention. Many imagery tasks require considerable time to complete, and thus the image must be maintained. Maintaining an imaged pattern requires effort, and only a limited amount of information can be visualized at the same time. This aspect of imagery has received remarkably little study, however, and the available research merely serves to verify that such introspections are correct [for a review, see 13].

\section{Reasoning using imagery}

One reason why image transformations are important is that images are more than a means by which information is learned, stored and retrieved. They also play a key role in several types of reasoning.

Concrete and abstract reasoning. Imagery can play a role in two distinct types of reasoning. First, one can reason about perceptual properties themselves. For example, consider how you decide what is the best route to take to get to the airport at a specific time of day from where you work, or how you would decide whether a sofa seen in the store would 'fit' in your living room. In both cases, imagery is used to carry out a kind of 'mental simulation'. Second, imagery can be used in abstract reasoning. Indeed, imagery apparently played a role in some of the key discoveries in science [see 19]. For example, an image of snakes biting their own tails apparently led Kekule to infer the 
ring-structure of benzene. In this sort of reasoning images are used as symbols. Such reasoning occurs when one visualizes Venn Diagrams or lines with dots on them as aids to solving logic problems.

Using imagery to comprehend language. Another kind of reasoning involves the inferences one makes when comprehending language. Imagery is often contrasted with verbal abilities, but it is clear that the two faculties work together in many ways. We earlier noted that imagery can help one to learn new information, including verbal information. In addition, imagery can help one to comprehend verbal descriptions. This is most obvious when the description specifies visual or spatial information. For example, imagery is likely to be used when one understands a description of the arrangement of an apartment or the design of a complex machine.

\section{OVERVIEW}

The articles in this special issue span the range of research on imagery, and either speak directly to the brain bases of imagery or have direct implications for future neuropsychological research.

\section{Learning and memory}

We begin with an article that harvests the results of decades of research on the role of imagery in memory. Richardson turns to the use of imagery mnemonics as a way to assist patients with memory impairments (caused by brain damage or disease). $\mathrm{He}$ found that patients with less severe memory impairments benefited most from imagery training, but the effectiveness of training was not strongly related to the locus of brain damage or to the etiology of the disorder. More strongly motivated patients also did better, but intelligence, imagery ability, and education were not very important predictors of the degree of improvement. It is of great interest that brain-damaged patients who could use mnemonics effectively nevertheless may not use this strategy spontaneously.

The early work on the role of imagery in memory focused on the importance of concrete vs abstract materials, or on the role of organizational factors in memory. De Beni and Pazzaglia consider memory for different kinds of mental images, focusing specifically on the role of contextual and autobiographical variables. They asked normal subjects to visualize a series of items, and measured how quickly the subjects could form the images, how well they could later recall the items, and also assessed subjective ratings of image quality. The items were visualized in isolation or in a specific context (with three kinds of context), and De Beni and Pazzaglia particularly were interested in the possibility that images of one's own life experiences have a special status in memory. And in fact such images required the most time to generate, were recalled very well, and were very vivid. From a neuropsychological perspective, this work is interesting because it invites researchers to attempt to understand the importance of this particular kind of imagery. It is possible, for example, that the right hemisphere plays a special role in storing images of specific exemplars; does it also play a special role for autobiographical images? 


\section{Perception and action}

In some ways imagery may have the same relation to other processes that glasses have to the nose: Once certain properties of a nose were present (presumably to warm air, direct scents to different nostrils, and so on), they could be used to hold up glasses. Similarly, once specific properties of basic sensory and response mechanisms were in place, they could be recruited into imagery-which then in turn may have become a function in its own right, thereafter being the subject of natural selection.

Goldenberg, Müllbacher and Nowak report the case of a brain-damaged patient who had cortical blindness, but still had visual mental imagery. This patient denied that she was blind, and this belief could have been based on her confusing visual mental images for actual percepts. This patient is interesting in part because her primary visual cortex was almost totally lesioned; only a small portion of the cortex at the occipital tip of the left upper calcarine lip was spared. Thus, her imagery apparently did not depend on an intact area 17, but rather relied (at least in large part) on higher-level visual areas that were not damaged. The authors note that this patient's imagery could have been triggered by tactile or acoustic perception. Once her vision recovered in the portion of the field that was registered by the preserved cortex, nonvisual input no longer appeared to induce the illusion of seeing. These findings suggested that connections from nonvisual cortex play a complex role in evoking imagery.

Servos and Goodale also examine a patient who had spared imagery in the face of impaired perception. This patient had a severe visual form agnosia, but nevertheless was able to scan mental images to search for specific features and could generate novel images by combining images of previously encoded stimuli. This patient had been shown earlier to use information about shape to control skilled grasping movements, even though she could not use this information to categorize or characterize the form. These findings suggest that sensory input, imagery, and motor control mechanisms have distinct ways of accessing stored visual representations; it is interesting that imagery and motor access were preserved together in the face of impaired sensory access. This patient appeared to have damage primarily to areas 18 and 19; most of area 17 was intact, as well most extrastriate cortex. The results may indicate that pathways within areas 18 and 19 are not essential for visual mental imagery, but play a critical role in providing input to higher visual areas during perception.

The fact that both motor control mechanisms and imagery can access representations of shape even when perceptual input cannot is consistent with results summarized in the following paper by Annett. Annett focuses on the kind of motor imagery that is used when one mentally practices performing a specific action. Annett reviews theoretical and practical issues that bear on the subjective, behavioral, and physiological methods used to study motor imagery. He argues that motor imagery relies on mechanisms that serve both spatial and motoric functions, and that the highly distributed nature of motor control blurs the question of whether motor imagery is primarily perceptual or motoric in nature.

Jeannerod also explores the role of the motor system in imagery. His central idea is that motor images have the same properties as the corresponding actual motor representations. He presents much evidence that supports this hypothesis. For example, the timing of simulated movements respects the same constraints as that of actual movements. In addition, simply imagining that a limb is moving produces an increase in specific reflexes in that limb, as occurs during actual movement. Moreover, imaging movement produces 
changes in cortex like those that occur when one actually executes an action. Jeannerod proposes a hierarchical model of the organization of action that provides an account for the similarities between imagined movement and actual movement; this model provides the theoretical foundations for the effects of mental practice reviewed in the previous paper.

The final paper in this section addresses a different sort of motor imagery, which is not related to the manipulation of shape. Wilson, Smith and Reisbert examine the role of subvocalization in auditory imagery. They examine the 'partnership' between the 'inner ear' and 'inner voice', and find that the two functions usually-but not always-work together. Although this paper is not explicitly neuropsychological in its orientation, it provides a beacon for further research; it seems clear that auditory imagery can be more deeply understood by adopting a parallel approach to that now used to study visual imagery. Indeed, it may turn out that the parallels run deeper than this; the 'inner ear' and visual 'image inspection' mechanisms may be functionally similar, and the 'inner voice' and some forms of visual 'image generation' mechanisms (particularly those in which eye movements can prompt images in a sequence) may be functionally similar. Such mechanisms are discussed in the articles that address the role of imagery in information processing.

\section{Information processing}

Enormous progress has been made recently in understanding the brain-bases of the mechanisms that generate visual mental images. Farah reviews recent evidence that speaks to two general issues about image generation. First, she considers the question of whether a distinct component process is used to generate visual mental images, or whether mechanisms that serve other purposes work together to accomplish this end. Second, she reviews findings that bear on the neuroanatomical localization of the processes that underlie image generation, and considers which regions of the brain are recruited by these processes. The following articles report additional findings that speak to these issues.

Stangalino, Semenza and Mondini report a detailed investigation of the effects of brain damage on the ability to generate visual mental images. They tested 70 unilaterally braindamaged patients with a set of tasks that was designed to assess distinct component processes. Consistent with the original retrospective analysis of Farah [7], these researchers found that damage to posterior portions of the left hemisphere most frequently were related to deficits in the ability to generate images.

Kosslyn, Hamilton, Maljkovic, Horwitz and Thompson provide evidence that visual mental images can be formed in two ways. One method relies on stored descriptions to arrange parts of image objects, and the other relies on stored information about metric distance to arrange parts. The former method is more effective when stimuli are presented in the right visual field (and hence are processed initially by the left cerebral hemisphere), whereas the other method is more effective when stimuli are presented in the left visual field (and hence are processed initially by the right cerebral hemisphere). According to their analysis, previous research with brain-damaged patients has used materials that cannot easily be visualized using the right-hemisphere processes, and hence patients with left-hemisphere damage have been selectively impaired.

Denis, Goncalves and Memmi focus on another aspect of image generation, namely its accuracy. In previous work, this group has shown that people can use verbal descriptions to construct images, which can then be scanned. Because time is required to scan each 
increment of distance, scan time can be used as an index of how accurately the parts of the image are positioned. Using this technique, Denis and his colleagues have shown that the structure of the initial description affects how accurately an image can be generated. But even when subjects are given poorly structured descriptions, their images improve after they see the description repeatedly. The present article reports a quantitative model and a computer simulation that accounts for this increase in image accuracy. In addition, new data from humans are reported that bear on this account, and underline the importance of 'regions of uncertainty' (i.e. indeterminancies about the locations of parts) in the image generation process.

Georgopolous and Pellizzer consider another aspect of information processing in imagery, mental rotation. They compare the processes that underlie mental rotation to those used to scan through memorized lists of digits. This paper integrates previous work by this group with monkeys and recent work with humans. The monkey research relied on single-cell recordings, and showed that motor processes are involved in at least some forms of mental rotation. The present work shows that mental rotation is in fact distinct from memory scanning in humans, which is consistent with the role of the motor system in imagery.

\section{Reasoning}

Vecchi, Monticelli and Cornoldi examine one of the key mechanisms that allows us to use imagery in reasoning: working memory. A major bottleneck in using imagery in reasoning is the capacity of working memory, and these researchers hypothesize that this capacity hinges on properties of a passive store and properties of active imagery operations. To test these ideas, subjects were asked to visualize moving through twodimensional and three-dimensional matrices; the matrices had some filled cells, and the subjects had to retain the image in order to perform the task. Experiments with congenitally blind people and with sighted people revealed that image capacity is a result of at least two different factors.

Geminiani, Bisiach, Berti and Rusconi argue that imagery plays such a central role in thinking that language cannot entirely compensate for severe impairment of 'analogue representations'. They argue that language does not have an independent role in thought, and provide suggestive evidence that syntactic structures themselves can be represented in a non-linguistic medium. This work is intriguing, and moves into an area that has received very little attention heretofor.

\section{Afterword}

We finish with some reflections by Cooper on "what counts as a mental image". After the wide and varied writings that have come before, this question gains force. Issues like this can only be addressed in the context of empirical findings, and can help not only to make sense of those findings but to guide one to consider additional research questions.

\section{REFERENCES}

1. Anderson, J. R. Arguments concerning representations for mental imagery. Psychol. Rev. 85, 249-277, 1978.

2. Baylor, G. W. A Treatise on the Mind's Eye. (Vol. Ed.) (Sec. Ed.). Carnegie Mellon University, Pittsburgh, Pennsylvania, 1971.

3. Behrmann, M., Winocur, G. and Moscovitch, M. Dissociation between mental imagery and object recognition in a brain-damaged patient. Nature 359, 636-637, 1992. 
4. Bower, G. H. Mental imagery and associative learning. In Cognition in Learning and Memory, L. Gregg (Editor), pp. 51-88. Wiley, New York, 1972.

5. Craver-Lemley, C. and Reeves, A. Visual imagery selectively reduces vernier acuity. Perception 16, 533-614, 1987.

6. Deutsch, G., Bourbon, W. T., Papanicolaou, A. C. and Eisenberg, H. Visuospatial experiments compared via activation of regional cerebral blood flow. Neuropsychologia 26, 445-452, 1988.

7. Farah, M. J. The neurological basis of mental imagery: A componential analysis. Cognition 18, 245-272, 1984.

8. Felleman, D. J. and Van Essen, D. C. Distributed hierarchical processing in primate cerebral cortex. Cerebr. Cortex 1, 1-47, 1991.

9. Finke, R. A. and Shepard, R. N. Visual functions of mental imagery. In Handbook of Perception and Human Performance, K. R. Boff, L. Kaufman and J. P. Thomas (Editors), pp. 37-1-37-55. Wiley-Interscience, New York, 1986.

10. Gardner, H. The Mind's New Science: A History of the Cognitive Revolution. Basic Books, New York, 1985.

11. Georgopolous, A. P., Lurito, J. T., Petrides, M., Schwartz, A. B. and Massey, J. T. Mental rotation of the neuronal population vector. Science 243, 234-236, 1989.

12. Kosslyn, S. M. Information representation in visual images. Cognit. Psychol. 7, 341-370, 1975.

13. Kosslyn, S. M. Image and Brain: The Resolution of the Imagery Debate. MIT Press, Cambridge, Mass., 1994.

14. Kosslyn, S. M. and Jolicoeur, P. A theory-based approach to the study of individual differences in mental imagery. In Aptitude, Learning and Instruction: Cognitive Processes Analysis of Aptitude, R. E. Snow, P. A. Federico and W. E. Montague (Editors). Lawrence Erlbaum, Hillsdale, New Jersey, 1981.

15. Levine, D. N., Warach, J. and Farah, M. J. Two visual systems in mental imagery: Dissociation of 'what' and 'where' in imagery disorders due to bilateral posterior cerebral lesions. Neurology 35, 1010-1018, 1985.

16. Paivio, A. Imagery and Verbal Processes. Holt, Rinehart \& Winston, New York, 1971.

17. Richardson, A. Mental practice: A review and discussion (Part I and II). Res. Quart. 38, 95-107; 263-273, 1967.

18. Segal, S. J. and Fusella, V. Influence of imaged pictures and sounds on detection of visual and auditory signals. J. Exp. Psychol. 83, 458-464, 1970.

19. Shepard, R. N. and Cooper, L. A. Mental Images and their Transformations. MIT Press, Cambridge, Mass., 1982.

20. Shepard, R. N. and Feng, C. A chronometric study of mental paper folding. Cognit. Psychol. 3, 228-243, 1972.

21. Shepard, R. N. and Metzler, J. Mental rotation of three-dimensional objects. Science 171, 701-703, 1971. 